\title{
The predictive value of routine laboratory tests for colorectal polyps: a retrospective study
}

\author{
Xinyi Feng ${ }^{1 \#}$, Xiuping Jiao ${ }^{2 \#}$, Yemin $\mathrm{Xu}^{1} \wedge$, Xi Xu${ }^{1} \wedge$, Yan $\mathrm{Zhu}^{3} \wedge$, Qiang She ${ }^{1 \wedge}$, Yaoyao $\mathrm{Li}^{1}$, Guiqing $\mathrm{Li}^{1}$, \\ Jian $\mathrm{Wu}^{1} \wedge$, Weiming Xiao ${ }^{1} \wedge$, Yanbing Ding $^{1}{ }^{\wedge}$, Bin Deng $^{1 \wedge}$ \\ ${ }^{1}$ Department of Gastroenterology, Affiliated Hospital of Yangzhou University, Yangzhou, China; ${ }^{2}$ Department of Health Management Centre, \\ Affiliated Hospital of Yangzhou University, Yangzhou, China; ${ }^{3}$ School of Medicine of Yangzhou University, Yangzhou, China \\ Contributions: (I) Conception and design: X Feng, X Jiao, B Deng; (II) Administrative support: W Xiao, Y Ding; (III) Provision of study materials or \\ patients: X Jiao, B Deng; (IV) Collection and assembly of data: X Feng, Y Xu, X Xu; (V) Data analysis and interpretation: X Feng, Y Zhu, Y Li, Q \\ She, G Li; (VI) Manuscript writing: All authors; (VII) Final approval of manuscript: All authors. \\ \#These authors contributed equally to this work. \\ Correspondence to: Bin Deng. Department of Gastroenterology, Affiliated Hospital of Yangzhou University, 368 Hanjiang Middle Road, Yangzhou \\ 225000, China. Email: chinadbin@126.com.
}

\begin{abstract}
Background: Colorectal cancer (CRC) has become the malignant tumor of the digestive tract with the highest incidence in our country, posing a serious threat to the health of our people. Early colon cancer is mostly due to the malignant transformation of colon polyps, so that early detection and resection have been shown to be effective in reducing the incidence and mortality of CRC. This study tried to investigate the related risk factors of and construct a predictive nomogram for colorectal polyps, providing meaningful guidance basis for risk stratification and screening.
\end{abstract}

Methods: A total of 1,799 patients who underwent colonoscopies in the Health Management Centre of the Affiliated Hospital of Yangzhou University were recruited to this study. Univariate and multivariate logistic analyses were performed to determine the risk factors for colorectal polyps, and a predictive nomogram was constructed based on the multivariable model. We determined the predictive value of the nomogram by receiver operating characteristic (ROC) curve, calibration curve, and decision curve analyses (DCAs).

Results: The logistic regression analysis showed that age $(\mathrm{P}<0.001)$, gender $(\mathrm{P}<0.001)$, eosinophil count $(\mathrm{P}=0.005)$, hemoglobin level $(\mathrm{P}=0.039)$, and LDL-C/HDL-C ratio $(\mathrm{LHR} ; \mathrm{P}<0.001)$ were independent predictors of the development of colorectal polyps. The above independent risk factors were incorporated, and an individualized nomogram model was successfully established. The C-index of the nomogram was 0.679 in our model, and with the bootstrap method, the prediction curve fit well with the ideal curve, suggesting that the prediction curve constructed in this study has good predictive ability.

Conclusions: Age, gender, eosinophil count, hemoglobin level, and LHR are risk factors for the development of colorectal polyps. Establishing a nomogram prediction model for colorectal polyps is helpful for the early clinical screening of high-risk patients with colorectal polyps, improving the detection rate of polyps and reducing the incidence of colorectal cancer (CRC).

Keywords: Colorectal polyps; risk factors; predictive model; nomogram

Submitted Dec 08, 2021. Accepted for publication Jan 30, 2022.

doi: 10.21037/jgo-21-933

View this article at: https://dx.doi.org/10.21037/jgo-21-933

^ ORCID: Xinyi Feng, 0000-0001-5960-1028; Yemin Xu, 0000-0002-9058-1966; Xi Xu, 0000-0003-1810-7220; Yan Zhu, 0000-0003-45642755; Qiang She, 0000-0001-8861-2674; Jian Wu, 0000-0003-0880-8787; Weiming Xiao, 0000-0002-8893-9076; Yanbing Ding, 00000002-0689-2199; Bin Deng, 0000-0002-5590-3755. 


\section{Introduction}

Colorectal cancer (CRC) is a prevalent cancer, and its incidence and mortality rank third and second, respectively, among cancers worldwide (1). It develops through 3 distinctive pathways, including the adenoma-carcinoma sequence, serrated pathway, and inflammatory pathway (2). The adenoma-carcinoma sequence is a classic pathway that explains the majority of sporadic CRC, and adenomatous polyps are thought to be the precursor lesions for the majority of CRC cases. It is estimated that approximately $85-90 \%$ of sporadic CRCs evolved from adenomas, but less than $10 \%$ of adenomas progressed to CRC (3). In this pathway, inactivating mutations in adenomatous polyposis coli (APC) gene result in overactivation of the Wnt/ $\beta$-catenin signalling pathway, triggering dysregulated cell proliferation and adenoma development (4). Turano et al. have shown that chronic inflammation can activate the Wnt/ $\beta$-catenin pathways by IL-6 signaling. Serrated polyps are precursors to approximately $10-15 \%$ of sporadic CRC (5). In the serrated pathway, it is suggested that some hyperplastic polyps, sessile serrated adenomas and traditional serrated adenomas may have malignant potential (6). In addition, some asymptomatic polyps may develop into malignancies that are more likely to be overlooked (7). Therefore, the early detection and resection of colorectal polyps is a critical way to reduce the incidence and mortality of CRC (8).

It takes at least 10 years for colorectal polyps to progress to CRC, leaving a large window of opportunity for the secondary prevention of CRC (2). Colonoscopy is now widely used to detect and remove polyps. However, colonoscopy is an invasive and relatively highcost procedure and carries risks associated with bowel preparation, sedation, bowel perforation, and bleeding $(9,10)$. These limitations contribute to low compliance and a low participation rate with colonoscopy screening in the general population (11). Therefore, it is more feasible for high-risk groups to undergo colonoscopy screening.

Previous studies have reported the relationship between living habits, demographic characteristics, and other factors and colorectal polyps, but there have been scarce studies discussing the association of routine laboratory tests and colorectal polyps (12-14). Routine laboratory tests are basic procedures in health examinations and have the advantages of wide popularity, high acceptance, and non-invasiveness. The aim of this study was to determine the risk factors for and evaluate the predictive values of colorectal polyps to improve the detection rate of colorectal precancerous lesions and provide supporting data for the subsequent screening of high-risk groups based on the results of multiple experimental examinations. We present the following article in accordance with the TRIPOD reporting checklist (available at https://jgo.amegroups.com/article/ view/10.21037/jgo-21-933/rc).

\section{Methods}

\section{Study population}

We examined the medical records of all participants who had colonoscopies between January 2017 and June 2021 in the Health Management Centre of the Affiliated Hospital of Yangzhou University. The following patients were excluded from our analysis: cases complicated with serious diseases (such as infection, chronic vital organ failure, and autoimmune diseases); cases complicated with intestinal diseases such as inflammatory bowel disease and CRC; cases with incomplete colonoscopies or incomplete clinical information; and cases with previous colorectal resection. Finally, a total of 1,799 participants were enrolled in this retrospective study. All procedures were carried out in compliance with the Helsinki Declaration (as revised in 2013). This study was approved by the Ethics Committee of the Affiliated Hospital of Yangzhou University (No. 2021-YKL06-09-004). The need for informed consent was waived due to the retrospective nature of this study.

\section{Data collection}

Clinical characteristics were obtained from the hospital medical database records with the assistance of the health management center and information center, without revealing personal information. Height and weight were measured on standardized machines. Body mass index (BMI) was calculated as weight divided by height squared $\left(\mathrm{kg} / \mathrm{m}^{2}\right)$. Waist circumferences were measured using a measuring tape at the smallest horizontal circumference between the costal margin and iliac crests. Smoking history, drinking habits, and comorbidities, such as hypertension and diabetes, were also recorded.

Blood samples were analyzed in the same laboratory. Routine laboratory tests included routine hematology, biochemistry, and lipid tests. In blood tests, the following data were collected: white blood cell (WBC) count, hemoglobin level, platelet level, eosinophil count, albumin level, serum creatinine (Scr) levels, uric acid (UA) level, total cholesterol (TC) level, triglyceride (TG) level, highdensity lipoprotein cholesterol (HDL-C) level, and low- 
density lipoprotein cholesterol (LDL-C) level. The TG/ HDL-C ratio (THR) was calculated by dividing the TG level by the HDL-C level, and the LDL-C/HDL-C ratio (LHR) was calculated by dividing the LDL-C level by the HDL-C level.

All colonoscopies were carried out by experienced endoscopists who performed more than 1,000 endoscopies annually. Biopsies were taken from lesions and 2 pathologists made pathological diagnosis. If the diagnostic result was controversial, the higher grade of the disease was named.

\section{Statistical analysis}

Continuous variables were defined as the mean \pm standard deviation, and categorical variables were given as percentages. Student's $t$-test was used for continuous variables, and the chi-square test was used for categorical variables. Univariate and multivariate logistic regression were used to evaluate the factors associated with the presence of colorectal polyps.

According to the multivariate logistic regression analysis, we constructed a predictive nomogram model on the "rms" package of the R software. The nomogram is based on proportionally converting each regression coefficient in multivariate analyses to a 0 -point to 100 -point scale. The points are added according to independent variables to derive total points, which are converted to predicted incidences of colorectal polyps. We drew the receiver operating characteristic (ROC) curve and used Harrell's concordance index (C-index) to assess the discrimination ability of the developed nomogram. A C-index of 0.5 indicated poor discrimination ability, and 1.0 indicated excellent discrimination ability (15). For calibration, the nomogrampredicted probabilities were contrasted with the actual probabilities by bootstrapping with 1,000 resamples, and we calculated the corrected C-index. Decision curve analyses (DCAs) were performed to assess the clinical usefulness of the nomogram. Any $\mathrm{P}$ value $<0.05$ was considered statistically significant. The statistical analyses were conducted using SPSS 26.0 (IBM Corp., Chicago, IL, USA) and R software (version 4.1.0; https://www.r-project.org/).

\section{Results}

\section{Study population characteristics}

In total, 1,799 participants were included in the present study, among whom 853 (47.4\%) had colorectal polyps, and the remaining 946 (52.6\%) were classified as healthy controls. Among the participants with colorectal polyps, $421(49.4 \%)$ were identified as having neoplastic polyps (416 adenomatous polyps with or without intraepithelial neoplasia, 4 sessile serrated polyps, 1 serrated adenomas), and $432(50.6 \%)$ were identified as having nonneoplastic polyps (413 simple polyps, 19 hyperplastic polyps). Among the participants with adenomas, 63 were identified as having tubular adenomas, and 12 were identified as having tubulovillous adenomas. There were 647 participants with polyps in the left colon, 149 participants with polyps more than $1 \mathrm{~cm}$ in size, and 506 participants with more than 1 polyp.

The baseline characteristics of both groups are summarized in Table 1. Colorectal polyps were more frequently observed in participants who were older, male, with a smoking history and drinking habit, a higher BMI, and a higher waist circumference $(\mathrm{P}<0.05)$. Regarding complications, a higher proportion of hypertension was found in participants with colorectal polyps $(\mathrm{P}<0.05)$, while there was no difference in diabetes between the 2 groups $(\mathrm{P}=0.161)$. Regarding laboratory indicators, WBC counts, hemoglobin levels, eosinophil counts, albumin levels, Scr levels, UA levels, HDL-C levels, LDL-C levels, THR, and LHR changed significantly $(\mathrm{P}<0.05)$ in the participants with colorectal polyps, whereas platelet, TC, and TG levels were not significantly different between the 2 groups $(\mathrm{P}>0.05)$.

\section{Independent risk factors for colorectal polyps}

Binary logistic regression analysis was applied to evaluate the factors associated with the presence of colorectal polyps. Univariate analysis revealed that age, gender, BMI, waist circumference, smoking, drinking, hypertensive disease, WBC count, hemoglobin level, eosinophil count, albumin level, Scr level, UA level, HDL-C level, LDL-C level, and LHR were related to colorectal polyps $(\mathrm{P}<0.05)$ (Table 2). According to collinearity diagnosis, we found that the variance inflation factor (VIF) of all the variables except LDL-C levels were $<10$. Then, all the variables, except LDL-C levels, in the univariate analysis were further processed by multivariate analysis (Table 2). The results indicated that age $(\mathrm{P}<0.001)$, gender $(\mathrm{P}<0.001)$, eosinophil count $(\mathrm{P}=0.005)$, hemoglobin level $(\mathrm{P}=0.039)$, and LHR $(\mathrm{P}<0.001)$ were independent risk factors for colorectal polyps.

Based on the above results, the predictive equation can be described as riskindex $=-5.879+0.554 \times($ gender $)+0.058$ $\times($ age $)+1.178 \times($ eosinophil count $)+0.011 \times($ hemoglobin 
Table 1 Clinical and laboratory characteristics of the participants

\begin{tabular}{|c|c|c|c|}
\hline Variables & Participants without colorectal polyps $(n=946)$ & Participants with colorectal polyps $(n=853)$ & $P$ value \\
\hline Age, years & $49 \pm 9.00$ & $53 \pm 8.67$ & $<0.001$ \\
\hline $\mathrm{BMI}, \mathrm{kg} / \mathrm{m}^{2}$ & $24.62 \pm 3.05$ & $25.19 \pm 2.66$ & $<0.001$ \\
\hline Waist circumference, $\mathrm{cm}$ & $86.57 \pm 6.68$ & $88.13 \pm 7.44$ & $<0.001$ \\
\hline Drinking (\%) & $282(29.8)$ & $331(38.8)$ & $<0.001$ \\
\hline Hypertension (\%) & $234(24.7)$ & $249(29.2)$ & 0.033 \\
\hline Diabetes (\%) & $98(10.4)$ & $106(12.4)$ & 0.167 \\
\hline WBC count, $\times 10^{9} / \mathrm{L}$ & $6.08 \pm 1.42$ & $6.24 \pm 1.64$ & 0.026 \\
\hline Eosinophil count, $\times 10^{9} / \mathrm{L}$ & $0.15 \pm 0.11$ & $0.18 \pm 0.14$ & $<0.001$ \\
\hline Albumin level, $\mathrm{g} / \mathrm{L}$ & $43.66 \pm 2.09$ & $43.46 \pm 2.06$ & 0.048 \\
\hline Scr level, $\mu \mathrm{mol} / \mathrm{L}$ & $75.77 \pm 14.85$ & $79.03 \pm 14.66$ & $<0.001$ \\
\hline UA level, $\mu \mathrm{mol} / \mathrm{L}$ & $342.73 \pm 81.03$ & $360.20 \pm 73.91$ & $<0.001$ \\
\hline TC level, mmol/L & $4.89 \pm 0.92$ & $4.94 \pm 0.87$ & 0.227 \\
\hline TG level, mmol/L & $1.96 \pm 1.95$ & $2.10 \pm 1.65$ & 0.110 \\
\hline HDL-C level, mmol/L & $1.30 \pm 0.30$ & $1.23 \pm 0.29$ & $<0.001$ \\
\hline LDL-C level, mmol/L & $2.68 \pm 0.74$ & $2.76 \pm 0.72$ & 0.024 \\
\hline
\end{tabular}

Data are presented as $\mathrm{n}(\%)$ or mean \pm standard deviation. BMI, body mass index; Scr, serum creatinine; UA, uric acid; TC, total cholesterol; TG, triglyceride; HDL-C, high density lipoprotein-cholesterol; LDL-C, low density lipoprotein-cholesterol; THR, TG/HDL-C, LHR, LDL-C/HDL-C.

level) $+0.297 \times($ LHR $)$.

\section{Building and validating a predictive nomogram model}

The predictive nomogram relying on identified risk factors from multivariate logistic regression models is shown in Figure 1. Each variable was assigned a score, and the total number of points could be associated with the probability of colorectal polyps by calculating every point of the variables. Then, the ROC curve of the nomogram was established (Figure $2 A$ ). The $\mathrm{C}$-index of the nomogram was $0.679[95 \%$ confidence interval (CI): 0.654 to 0.703 ] in our model and 0.675 by bootstrapping analysis, suggesting that the model had good discriminative ability. The calibration curve of the nomogram showed that the agreement between predicted and observed probability was great (Figure $2 B$ ). In addition, DCAs showed that the predictive model had significant net benefits when the threshold probabilities were between 0.2 and 0.7 , demonstrating the potential clinical benefit of the predictive model (Figure 2C).

\section{Discussion}

In this study, we found that age, gender, BMI, smoking, waist circumference, drinking, hypertensive disease, WBC count, hemoglobin level, eosinophil count, albumin level, Scr level, UA level, HDL-C level, LDL-C level, and LHR were related to colorectal polyps $(\mathrm{P}<0.05)$, and age, gender, 
Table 2 Univariate and multivariate analysis of factors for predicting colorectal polyps

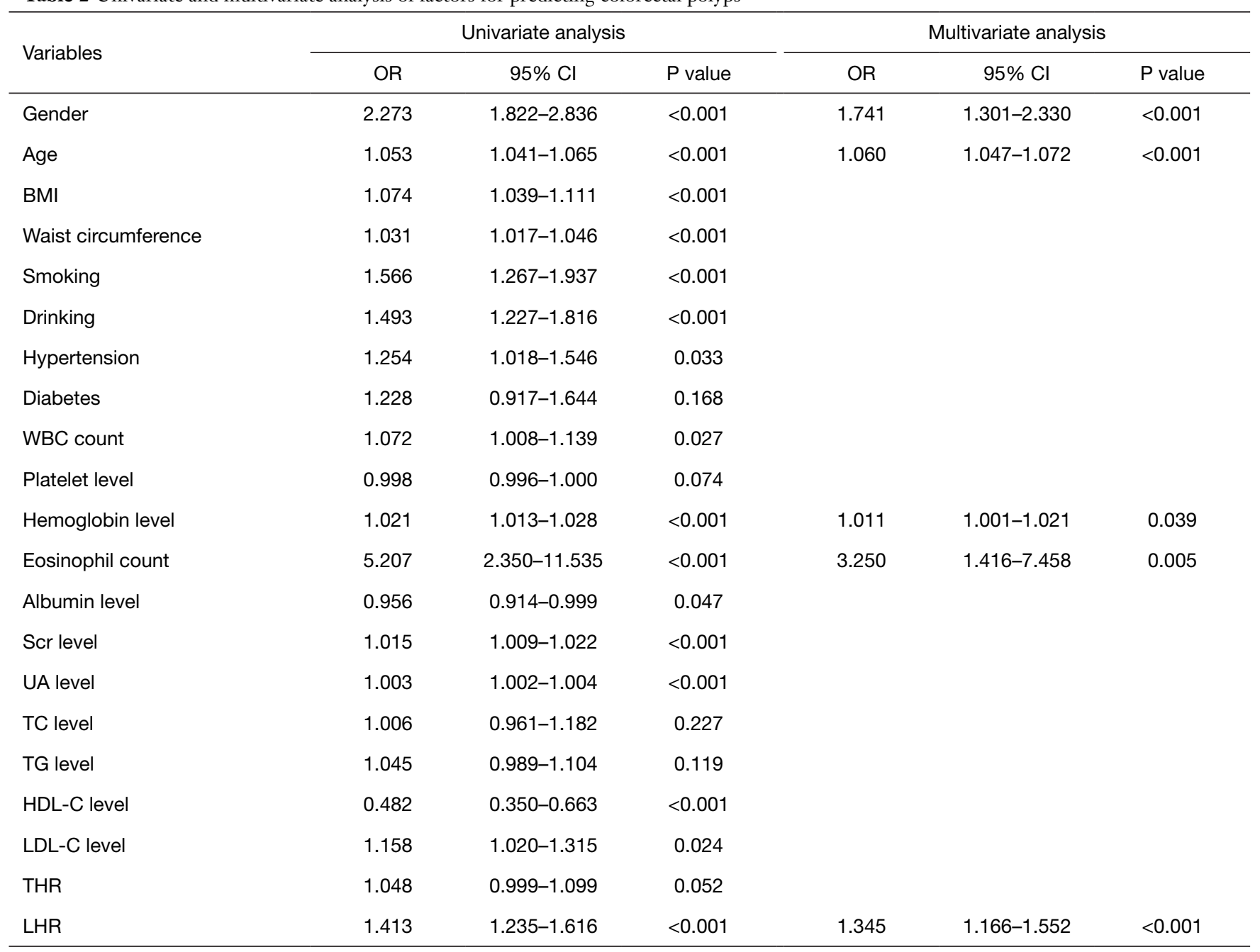

OR, odds ratio; Cl, confidence interval; BMI, body mass index; WBC, white blood cell; Scr, serum creatinine; UA, uric acid; TC, total cholesterol; TG, triglyceride; HDL-C, high-density lipoprotein cholesterol; LDL-C, low-density lipoprotein cholesterol; THR, TG/HDL-C; LHR, LDL-C/HDL-C.

eosinophil counts, hemoglobin levels, and LHR were independent risk factors for colorectal polyps. Moreover, we established a diagnostic nomogram based on laboratory indicators for predicting colorectal polyps, and the $\mathrm{C}$-index was 0.679 .

A positive association between age and the risk of colorectal polyps has been found in most studies. Populationbased national cancer screening programmes have shown that the detection rates for advanced neoplasms, non-advanced adenomas, and any neoplasms increase with age (14). This study also found that age is an independent risk factor for colorectal polyps. However, the incidence among younger patients under the age of 50 is increasing (16). Future studies need to increase the sample size and stratify the age to study the correlation of age with polyps.

Studies have indicated that the incidence of colorectal polyps in men is almost twice that in women $(17,18)$. One of the reasons is that men were more likely to be screened by colonoscopy than women (19). This is consistent with our research results. Computed tomography colonography (CTC), fecal immunochemical testing (FIT), and multitarget stool DNA testing may improve compliance for screening in women due to their convenience and non-invasiveness (20-22). Regarding the sensitivity and specificity of these tests, many trials should be conducted to support their reliability. 


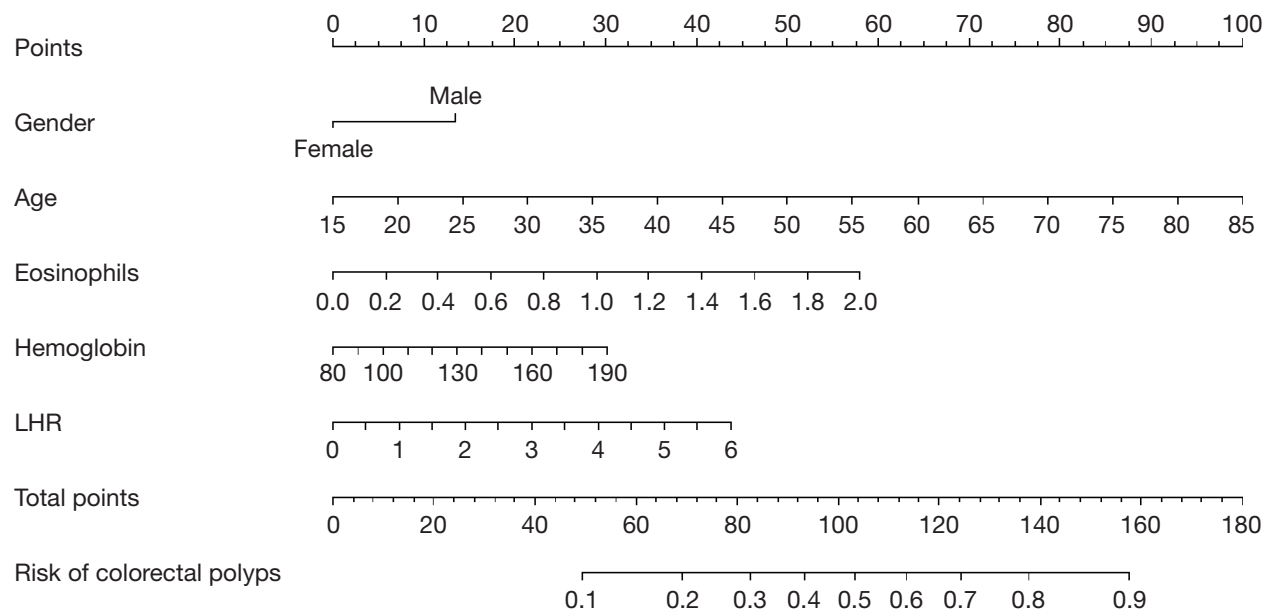

Figure 1 A nomogram for predicting colorectal polyps. LHR, LDL-C/HDL-C ratio; LDL-C, low-density lipoprotein cholesterol; HDL-C, high-density lipoprotein cholesterol.
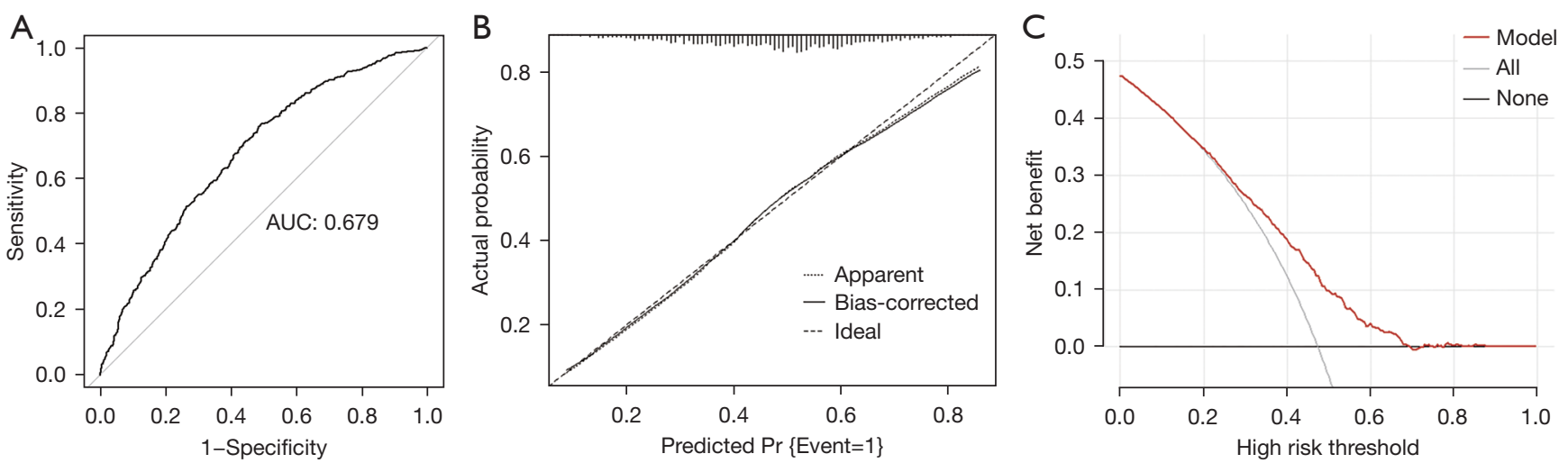

Figure 2 Evaluation of the nomogram model. (A) ROC curve of the nomogram for predicting colorectal polyps. (B) Calibration curves of the nomogram for predicting colorectal polyps. The predicted colorectal polyp risk is shown on the $\mathrm{X}$-axis. The actual diagnosed colorectal polyps is shown on the Y-axis. The $45^{\circ}$ line indicates location of the ideal nomogram. Broken line indicates actual nomogram performance. (C) Decision curve analysis of the nomogram for predicting colorectal, demonstrating the net benefit associated with use of the nomogram for the prediction of colorectal polyps. ROC, receiver operating characteristic.

Eosinophils are key immune effectors and inflammatory cells and play an important role in diseases such as tumors, chronic obstructive pulmonary disease (COPD), and coronary artery disease (CAD). Previous studies have mostly explored the relationship between eosinophils and colorectal cancer (CRC) (23). In our study, we found that eosinophil counts were higher in participants with colorectal polyps and had predictive value for polyps, which was in accordance with a previous study (24). A possible mechanisms is that the inflammatory response initiates the recruitment of eosinophils (25). However, specific mechanisms need to be further explored.

Hemoglobin is a special protein that transports oxygen in red blood cells. Previous studies have revealed that an elevated hemoglobin level increases the risk of nonalcoholic fatty liver disease and metabolic syndrome, which are associated with colorectal polyps $(26,27)$. Shen et al. conducted a cross-sectional study to explore the relationship between hemoglobin levels and colorectal polyps, and they found that elevated hemoglobin levels were significantly and independently associated with the prevalence and risk of colorectal polyps in asymptomatic adults (28). Similar 
to the study mentioned above, elevated hemoglobin levels independently increased the risk of colorectal polyps in our study. There are several probable explanations. First, hemoglobin might upregulate miR-144 expression, leading to an inflammatory response in microglia (29). In addition, hemoglobin, which is an iron-containing metalloprotein, might cause iron-induced oxidative stress (30). These processes could increase the possibility that colon adenomas transform into tumors via the adenoma-carcinoma sequence.

Recently, LHR has been shown to be a surrogate marker of insulin resistance and mixed dyslipidemia (31,32). Multiple studies have reported dyslipidemia is an independent risk factor for colorectal adenoma. Carot et al. reported that dyslipidemia increased the risk of new advanced lesions 3 years after polypectomy, and surveillance guideline strategies should be optimized (33). Xie et al. found that increased LDL-C and TG levels were correlated with the occurrence of polyps. The combination of the 2 serum indicators was useful to assess the risk of colorectal lesions (34). Although there was no significant difference in TC $(\mathrm{P}=0.227)$ and TG $(\mathrm{P}=0.11)$ levels in our study, we found that LHR was an independent predictor of colorectal polyps, which indirectly reflected the relationship between dyslipidemia and colorectal polyps. A possible explanation is that dyslipidemia strengthens the inflammatory response and accelerates injury to the mucosal epithelium, which has a great influence on the occurrence of tumors (35).

According to current studies, smoking is a modifiable risk factor for colorectal polyps (7,36,37). A study by Pan et al. showed that regular cigarette smoking is an independent risk factor for colorectal polyps in a Chinese population (7). According to a study by Lee et al., the colorectal polyp prevalence increases 3.40 times in current smokers compared to "never" smokers (37). For drinking, the current conclusions are different $(36,37)$. In our study, we failed to show the effect of smoking and alcohol consumption on the development of colorectal polyps. It may be that the lifestyle and genes of our participants are different from those of other regions. Multicenter research should be further carried out to explore the association between smoking, drinking and colorectal polyps.

Our study had several limitations. First, this was a retrospective single-center study, and we only enrolled participants in the health management center, which might have led to selection and recall bias. Therefore, a larger, multicenter prospective study is needed to validate the effectiveness of this model. Second, we failed to evaluate the risk factors for different pathological types of colorectal polyps. Third, we lacked some data, including the dietary habits and the family history of the examined population. These factors might also be associated with the development of colorectal polyps. Fourth, we only described the association of predictors with colorectal polyps, and the mechanisms of these factors in the development of colorectal polyps are expected to be studied in the future. Fifth, the model verification method only incorporated internal random verification. External validation is required through future studies.

In conclusion, our study indicated that age, gender, eosinophil count, hemoglobin level, and LHR were independent risk factors for colorectal polyps. We established an early predictive model based on laboratory indicators that could be quickly obtained on hospital admission. This model can be conveniently used to facilitate the prediction of the individual risk of colorectal polyps. These data provide support for the subsequent screening of high-risk groups with colorectal polyps to better prevent and manage this disease.

\section{Acknowledgments}

The authors thank all of the staff from the Department of Gastroenterology and Health Management Centre, Affiliated Hospital of Yangzhou University.

Funding: This work was supported by the Key Project for Social Development of Yangzhou (No. YZ2020069).

\section{Footnote}

Reporting Checklist: The authors have completed the TRIPOD reporting checklist. Available at https://jgo. amegroups.com/article/view/10.21037/jgo-21-933/rc

Data Sharing Statement: Available at https://jgo.amegroups. com/article/view/10.21037/jgo-21-933/dss

Conflicts of Interest: All authors have completed the ICMJE uniform disclosure form (available at https://jgo.amegroups. com/article/view/10.21037/jgo-21-933/coif). The authors have no conflicts of interest to declare.

Etbical Statement: The authors are accountable for all aspects of the work in ensuring that questions related to the accuracy or integrity of any part of the work are appropriately investigated and resolved. All procedures were 
carried out in compliance with the Helsinki Declaration (as revised in 2013). This study was approved by the Ethics Committee of the Affiliated Hospital of Yangzhou University (No. 2021-YKL06-09-004). The need for informed consent was waived due to the retrospective nature of this study.

Open Access Statement: This is an Open Access article distributed in accordance with the Creative Commons Attribution-NonCommercial-NoDerivs 4.0 International License (CC BY-NC-ND 4.0), which permits the noncommercial replication and distribution of the article with the strict proviso that no changes or edits are made and the original work is properly cited (including links to both the formal publication through the relevant DOI and the license). See: https://creativecommons.org/licenses/by-nc-nd/4.0/.

\section{References}

1. Sung H, Ferlay J, Siegel RL, et al. Global Cancer Statistics 2020: GLOBOCAN Estimates of Incidence and Mortality Worldwide for 36 Cancers in 185 Countries. CA Cancer J Clin 2021;71:209-49.

2. Keum N, Giovannucci E. Global burden of colorectal cancer: emerging trends, risk factors and prevention strategies. Nat Rev Gastroenterol Hepatol 2019;16:713-32.

3. Conteduca V, Sansonno D, Russi S, et al. Precancerous colorectal lesions (Review). Int J Oncol 2013;43:973-84.

4. Aceto GM, Catalano T, Curia MC. Molecular Aspects of Colorectal Adenomas: The Interplay among Microenvironment, Oxidative Stress, and Predisposition. Biomed Res Int $2020 ; 2020: 1726309$.

5. Turano M, Cammarota F, Duraturo F, et al. A Potential Role of IL-6/IL-6R in the Development and Management of Colon Cancer. Membranes (Basel) 2021;11:312.

6. Noffsinger AE. Serrated polyps and colorectal cancer: new pathway to malignancy. Annu Rev Pathol 2009;4:343-64.

7. Pan J, Cen L, Xu L, et al. Prevalence and risk factors for colorectal polyps in a Chinese population: a retrospective study. Sci Rep 2020;10:6974.

8. Zauber AG, Winawer SJ, O'Brien MJ, et al. Colonoscopic polypectomy and long-term prevention of colorectalcancer deaths. N Engl J Med 2012;366:687-96.

9. Levin B, Lieberman DA, McFarland B, et al. Screening and surveillance for the early detection of colorectal cancer and adenomatous polyps, 2008: a joint guideline from the American Cancer Society, the US Multi-Society Task Force on Colorectal Cancer, and the American College of
Radiology. Gastroenterology 2008;134:1570-95.

10. Cooper GS, Kou TD, Rex DK. Complications following colonoscopy with anesthesia assistance: a population-based analysis. JAMA Intern Med 2013;173:551-6.

11. Inadomi JM, Vijan S, Janz NK, et al. Adherence to colorectal cancer screening: a randomized clinical trial of competing strategies. Arch Intern Med 2012;172:575-82.

12. Gupta S, Jacobs ET, Baron JA, et al. Risk stratification of individuals with low-risk colorectal adenomas using clinical characteristics: a pooled analysis. Gut 2017;66:446-53.

13. Nakai K, Watari J, Tozawa K, et al. Sex differences in associations among metabolic syndrome, obesity, related biomarkers, and colorectal adenomatous polyp risk in a Japanese population. J Clin Biochem Nutr 2018;63:154-63.

14. Chen H, Li N, Ren J, et al. Participation and yield of a population-based colorectal cancer screening programme in China. Gut 2019;68:1450-7.

15. Balachandran VP, Gonen M, Smith JJ, et al. Nomograms in oncology: more than meets the eye. Lancet Oncol 2015;16:e173-80.

16. Chen Z, Hu J, Zheng Z, et al. Location of colorectal adenomas and serrated polyps in patients under age 50. Int J Colorectal Dis 2019;34:2201-4.

17. McCashland TM, Brand R, Lyden E, et al. Gender differences in colorectal polyps and tumors. Am J Gastroenterol 2001;96:882-6.

18. Brenner H, Altenhofen L, Stock C, et al. Incidence of colorectal adenomas: birth cohort analysis among 4.3 million participants of screening colonoscopy. Cancer Epidemiol Biomarkers Prev 2014;23:1920-7.

19. Etzioni DA, Ponce NA, Babey SH, et al. A populationbased study of colorectal cancer test use: results from the 2001 California Health Interview Survey. Cancer 2004;101:2523-32.

20. Chu LL, Weinstein S, Yee J. Colorectal cancer screening in women: an underutilized lifesaver. AJR Am J Roentgenol 2011;196:303-10.

21. Imperiale TF, Ransohoff DF, Itzkowitz SH, et al. Multitarget stool DNA testing for colorectal-cancer screening. N Engl J Med 2014;370:1287-97.

22. Li JN, Yuan SY. Fecal occult blood test in colorectal cancer screening. J Dig Dis 2019;20:62-4.

23. Loktionov A. Eosinophils in the gastrointestinal tract and their role in the pathogenesis of major colorectal disorders. World J Gastroenterol 2019;25:3503-26.

24. Moezzi J, Gopalswamy N, Haas RJ Jr, et al. Stromal 
eosinophilia in colonic epithelial neoplasms. Am J

Gastroenterol 2000;95:520-3.

25. Lotfi R, Lee JJ, Lotze MT. Eosinophilic granulocytes and damage-associated molecular pattern molecules (DAMPs): role in the inflammatory response within tumors. J Immunother 2007;30:16-28.

26. Chung GE, Yim JY, Kim D, et al. Associations between hemoglobin concentrations and the development of incidental metabolic syndrome or nonalcoholic fatty liver disease. Dig Liver Dis 2017;49:57-62.

27. He S, Gu H, Yang J, et al. Hemoglobin concentration is associated with the incidence of metabolic syndrome. BMC Endocr Disord 2021;21:53.

28. Shen C, Shi X, Al-Ashoor M, et al. Association between Hemoglobin Levels and Colorectal Polyps in Asymptomatic Chinese Adults. Gastroenterol Res Pract 2020;2020:3808163.

29. Wang Z, Yuan B, Fu F, et al. Hemoglobin enhances miRNA-144 expression and autophagic activation mediated inflammation of microglia via mTOR pathway. Sci Rep 2017;7:11861.

30. Ghamarchehreh ME, Jonaidi-Jafari N, Bigdeli M, et al. Iron Status and Metabolic Syndrome in Patients with Non-Alcoholic Fatty Liver Disease. Middle East J Dig Dis 2016;8:31-8.

31. Kawamoto R, Tabara Y, Kohara K, et al. Low-density lipoprotein cholesterol to high-density lipoprotein

Cite this article as: Feng $\mathrm{X}$, Jiao $\mathrm{X}, \mathrm{Xu} \mathrm{Y,} \mathrm{Xu} \mathrm{X,} \mathrm{Zhu} \mathrm{Y,} \mathrm{She}$ Q, Li Y, Li G, Wu J, Xiao W, Ding Y, Deng B. The predictive value of routine laboratory tests for colorectal polyps: a retrospective study. J Gastrointest Oncol 2022;13(1):256-264. doi: 10.21037/jgo-21-933 cholesterol ratio is the best surrogate marker for insulin resistance in non-obese Japanese adults. Lipids Health Dis 2010;9:138.

32. Wei L, Wei M, Chen L, et al. Low-density lipoprotein cholesterol : high-density lipoprotein cholesterol ratio is associated with incident diabetes in Chinese adults: A retrospective cohort study. J Diabetes Investig 2021;12:91-8.

33. Carot L, Navarro G, Naranjo-Hans D, et al. Predictors of Metachronous Risk Polyps After Index Colonoscopy. Clin Transl Gastroenterol 2021;12:e00304.

34. Xie C, Wen P, Su J, et al. Elevated serum triglyceride and low-density lipoprotein cholesterol promotes the formation of colorectal polyps. BMC Gastroenterol 2019;19:195.

35. Chen K, Guo J, Zhang T, et al. The Role of Dyslipidemia in Colitis-Associated Colorectal Cancer. J Oncol 2021;2021:6640384.

36. Øines M, Helsingen LM, Bretthauer M, et al. Epidemiology and risk factors of colorectal polyps. Best Pract Res Clin Gastroenterol 2017;31:419-24.

37. Lee K, Kim YH. Colorectal Polyp Prevalence According to Alcohol Consumption, Smoking and Obesity. Int J Environ Res Public Health 2020;17:2387.

(English Language Editor: J. Jones) 